\title{
Verzeichnis \\ der Abkürzungen und des Schrifttums
}

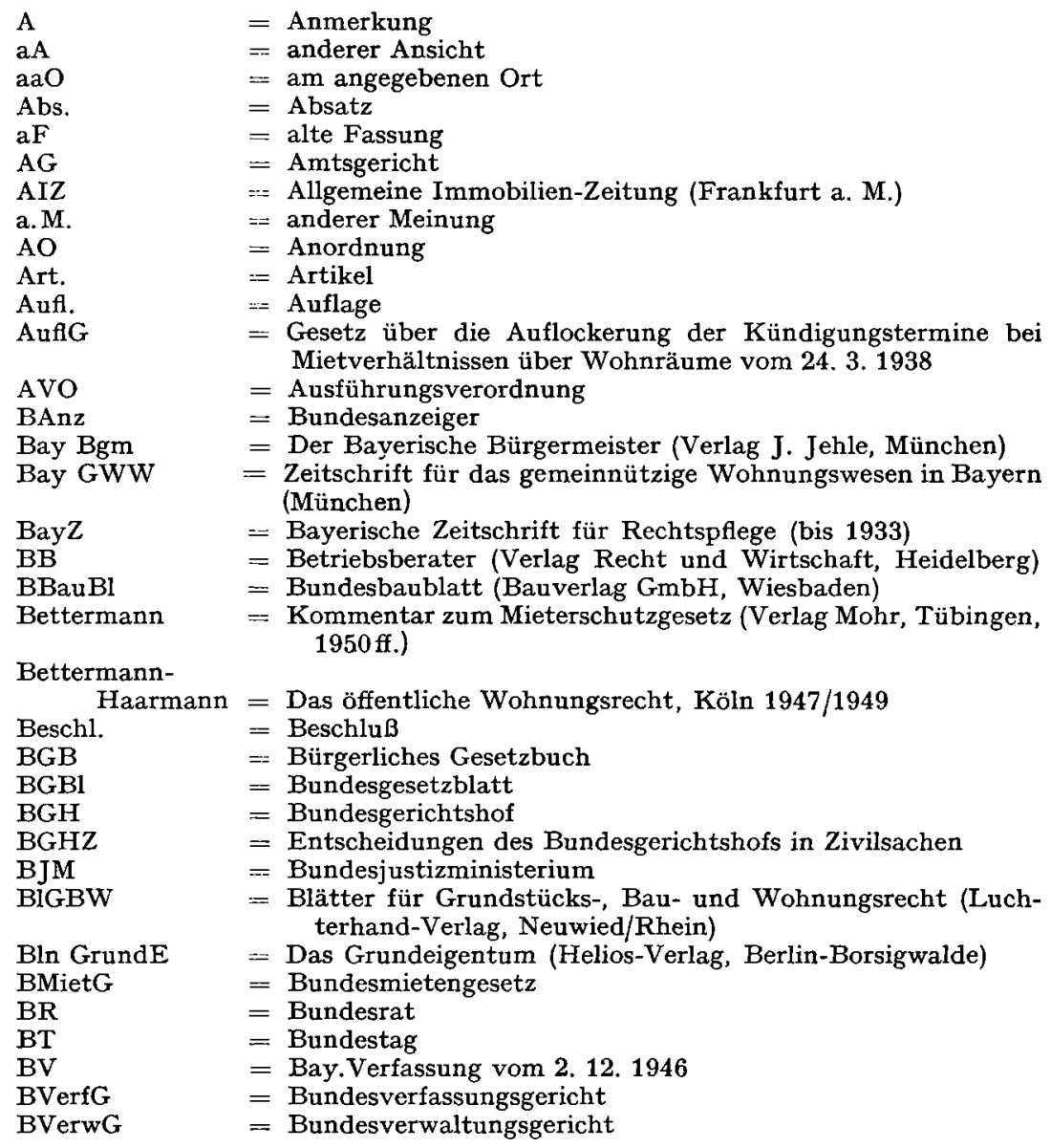




\section{Abkürzungen}

BVerwGE

DJ

DJZ

DMR

DÖV

Drews

DRZ

DRiZ

DVBl

DWW

DWohnA

EA

Ebel

Erl

ESVGH

FamRZ

FrWW

GG

Glaser

GKG

GRMG

Groothold

GS

GVBl

GWW

Hans

HGBR

Hbg GrundE

h.M.

HMR

HuW

i.d.F.

JMBl

JR

JZ

Kap.

KG

Kiefersauer-
= Entscheidungen des Bundesverwaltungsgerichts (Carl Heymanns Verlag, Berlin/Köln)

$=$ Deutsche Justiz

$=$ Deutsche Juristenzeitung (Verlag Mohr, Tübingen)

$=$ Deutsches Mietrecht

$=$ Die öffentliche Verwaltung (Verlag Kohlhammer, Stuttgart)

$=$ Erstes Bundesmietengesetz (Aschendorffsche Verlagsbuchhandlung, Münster)

$=$ Deutsche Rechtszeitschrift (Verlag Mohr, Tübingen)

$=$ Deutsche Richterzeitung (Verlag Heymanns, Berlin)

$=$ Deutsches Verwaltungsblatt (Verlag Heymanns, Berlin/Köln)

$=$ Deutsche Wohnungswirtschaft (Düsseldorf)

$=$ Deutsches Wohnungsarchiv

$=$ Einigungsamt und Mieteinigungsamt

= Kom. z. Mieterschutzgesetz, 8. Auf., Berlin 1938

$=\operatorname{Erla} B$

$=$ Entscheidungssammlung der Hess. und Württ.-Bad. Verwaltungsgerichtshöfe (Verlag Müller, Karlsruhe)

= Zeitschrift für das gesamte Familienrecht (Deutscher Heimatverlag, Bielefeld)

$=$ Die freie Wohnungswirtschaft (Verlag Hanseatisches Werbekontor Heuser \& Co., Hamburg)

$=$ Grundgesetz für die Bundesrepublik Deutschland

$=$ Entscheidungen aus dem Miet-, Wohnungs- und Grundstücksrecht (Verlag Haus und Grund, Köln)

$=$ Gerichtskostengesetz

$=$ Geschäftsraummietengesetz v. 25. 6. 1952

= Kommentar zum Ersten Bundesmietengesetz (Werner Verlag $\mathrm{GmbH}$, Düsseldorf)

$=$ Preuß. Gesetzsammlung

$=$ Gesetz- und Verordnungsblatt

$=$ Gemeinnütziges Wohnungswesen (Hammonia-Verlag, Hamburg)

$=$ Mieterschutzgesetz, 6. und 7. Aufl. (Münster 1956)

$=$ Handbuch des Grundstücks- und Baurechts (Werner-Verlag, $\mathrm{GmbH}$, Düsseldorf)

$=$ Hamburger Grundeigentum (Nölke-Verlag, Hamburg)

$=$ herrschende Meinung

$=$ Handbuch des gesamten Miet- und Raumrechts (WernerVerlag GmbH, Düsseldorf) bis 1951, fortgesetzt mit ZMR

$=$ Haus und Wohnung (Verlag Gubalke, Berlin) bis 30. 7. 1957

$=$ in der Fassung

$=$ Justizministerialblatt (mit Landeszusatz)

$=$ Juristische Rundschau (Verlag Walter de Gruyter \& Co., Berlin)

$=$ Juristenzeitung (Verlag Mohr, Tübingen)

$=$ Kapitel

= Kammergericht

Glaser $=$ Geschäftsraummiete (Schweitzer Verlag, Berlin u. München, 1956, 2. Aufl.) 


\section{Abkürzungen}

\begin{tabular}{|c|c|}
\hline Komm & $==$ Kommentar \\
\hline KO & $=$ Konkursordnung \\
\hline KündSchAusfVO & $\begin{array}{l}=\text { Ausführungsverordnung zur Verordnung über Kündigungs- } \\
\text { schutz für Miet- und Pachträume }\end{array}$ \\
\hline KündSchVo & $\begin{array}{l}=\text { Verordnung über Kündigungsschutz für Miet- und Pacht- } \\
\text { räume vom 28. 8. } 1937\end{array}$ \\
\hline LG & $=$ Landgericht \\
\hline LVG & $=$ Landesverwaltungsgericht \\
\hline MDR & $\begin{array}{c}=\begin{array}{c}\text { Monatsschrift für Deutsches Recht (Deutsche Rechtspre- } \\
\text { chungs-Verlags-GmbH, Hamburg) }\end{array}\end{array}$ \\
\hline MEA & $=$ Mieteinigungsamt \\
\hline MietenVO & $\begin{array}{l}=\text { Verordnung über die Miethöhe für neugeschaffenen Wohn- } \\
\text { raum vom 20.11.1950 (BGBI I, 759) }\end{array}$ \\
\hline MinBl. & $=$ Ministerialblatt \\
\hline MittB1 & $=$ Mitteilungsblatt \\
\hline MSchAusnVO & $\begin{array}{c}=\begin{array}{c}\text { Verordnung über Ausnahmen } \\
\text { 27. 11. 1951 (BGBl I, 926) }\end{array} \text { vom Mieterschutz vom } \\
\end{array}$ \\
\hline MSchG & $=$ Mieterschutzgesetz \\
\hline n.F. & $=$ neue Fassung \\
\hline NJW & $\begin{array}{l}=\text { Neue Juristische Wochenschrift (C. H. Becksche Verlags- } \\
\text { buchhandlung, München und Berlin) }\end{array}$ \\
\hline NHG & $=$ Niedersächs.Haus- und Grundbesitz (Hannover) \\
\hline Nds Rpfl & $=$ Niedersächs.Rechtspflege (Hannover) \\
\hline NRW & $=$ Nordrhein-Westfalen \\
\hline $\mathrm{OGH}$ & $=$ Oberster Gerichtshof für die Brit.Zone Köln \\
\hline OLG & $=$ Oberlandesgericht \\
\hline OVG & $=$ Oberverwaltungsgericht \\
\hline OVGE & $\begin{array}{l}=\text { Amtl Sammlung der Entscheidungen der Oberverwaltungs- } \\
\text { gerichte für das Land Nordrhein-Westfalen in Münster } \\
\text { sowie für die Länder Niedersachsen und Schleswig-Hoistein } \\
\text { in Lüneburg (Aschendorffsche Verlagsbuchhandlung, } \\
\text { Münster) }\end{array}$ \\
\hline PreisfreigabeAO & $\begin{array}{c}=\text { Anordnung über Preisbildung und Preisüberwachung nach } \\
\text { der Währungsreform vom 25.6.1948 (WiGBl. S. 61) }\end{array}$ \\
\hline PreisG & $\begin{array}{l}=\text { Ubergangsgesetz über Preisbildung und Preisüberwachung } \\
\text { vom 10.4. 1948 (WiGBl. S. 27) }\end{array}$ \\
\hline PStopVO & $\begin{array}{l}=\text { Verordnung über das Verbot von Preiserhöhungen vom } \\
\text { 26.11.1936 (RGBl I, 955) }\end{array}$ \\
\hline RAnz & $=$ Reichsanzeiger \\
\hline RArbBl & $=$ Reichsarbeitsblatt \\
\hline RdErl & $=$ Runderla $B$ \\
\hline $\mathrm{RdL}$ & $=$ Recht der Landwirtschaft \\
\hline RegBl & $=$ Regierungsblatt \\
\hline $\mathrm{RfPr}$ & $=$ Reichskommissar für die Preisbildung \\
\hline RG & $=$ Reichsgericht \\
\hline RGB1 & $=$ Reichsgesetzblatt \\
\hline $\mathrm{RGZ}$ & $\begin{array}{l}=\text { Entscheidungen des Reichsgerichts in Zivilsachen (Verlag } \\
\text { Walter de Gruyter \& Co., Berlin) }\end{array}$ \\
\hline RMietG & $=$ Reichsmietengesetz \\
\hline Roquette & $=$ Mietrecht, 4. Aufl. 1954 (Verlag Mohr, Tübingen) \\
\hline Roqu & $\begin{array}{l}=\text { Mieterschutzgesetz } 1956 \text { (C. H. Becksche Verlagsbuchhand- } \\
\text { lung, München und Berlin) }\end{array}$ \\
\hline
\end{tabular}




\section{Abkürzungen}

SchlHA = Schleswig-Holsteinischer Anzeiger - Justizministerialblatt des Landes Schleswig-Holstein (Schleswig)

Schopp-Groothold $=$ Kommentar zum Mieterschutzgesetz im Handbuch des gesamten Mietrechts (Werner-Verlag GmbH, Düsseldorf)

$\mathrm{SJZ}=$ Süddeutsche Juristenzeitung

Staudinger-

Kiefersauer = Kommentar zum BGB, 11. Aufl. 1955 (J. Schweitzer Verlag, München u. Berlin)

Stern $\quad=$ Mieterschutzgesetz, 10. Aufl., Berlin 1930

Urt. $\quad=$ Urteil

VersR = Versicherungsrecht (Karlsruhe)

Verw Rspr = Verwaltungsrechtsprechung in Deutschland (C. H. Becksche Verlagsbuchhandlung München und Berlin)

VG $\quad=$ Verwaltungsgericht

$\mathrm{VGH} \quad=$ Verwaltungsgerichtshof

VO $\quad=$ Verordnung

VOBl. $\quad=$ Verordnungsblatt

Vorbem = Vorbemerkung

WBewG = Wohnraumbewirtschaftungsgesetz

WEG $\quad=$ Gesetz über das Wohnungseigentum und das Dauerwohnrecht vom 15. 3. 1951 (BGBl I, 175)

WiGBl. = Gesetzblatt der Verwaltung des Vereinigten Wirtschaftsgebietes

WLVO = Verordnung zur Wohnraumlenkung vom 17. 2.1943 WM = Wohnungswirtschaft und Mietrecht (Strüdersche Buchdruk-

WMG $\quad=$ Wohnungsmangelgesetz

WohnBauG = Wohnungsbaugesetz

WohnG = Wohnungsgesetz

WVVO = Verordnung zur Wohnraumversorgung der luftkriegsbetroffenen Bevölkerung vom 21. 6. 1943

ZMR = Zeitschrift für Miet- und Raumrecht (Werner-Verlag GmbH, Düsseldorf)

$\mathrm{ZfV} \quad=$ Zeitschrift für Versicherungswesen (Allg. Fachverlag GmbH, Hamburg)

$\mathrm{ZPO} \quad=$ Zivilprozeßordnung

ZVG $\quad=$ Zwangsversteigerungsgesetz

$\mathrm{ZwVO} \quad=$ VO über das Verbot der $Z$ weckentfremdung von Wohnungen vom 14. 8. 1942 